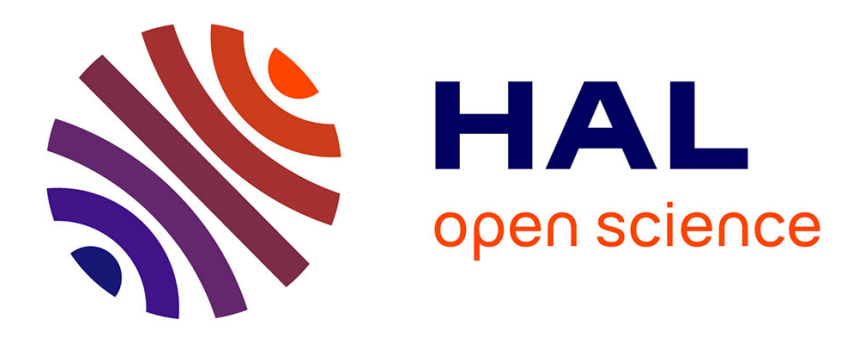

\title{
Unidirectional SPP excitation at asymmetrical two-layered metal gratings
}

\author{
A Roszkiewicz, W Nasalski
}

\section{To cite this version:}

A Roszkiewicz, W Nasalski. Unidirectional SPP excitation at asymmetrical two-layered metal gratings. Journal of Physics B: Atomic, Molecular and Optical Physics, 2010, 43 (18), pp.185401. 10.1088/09534075/43/18/185401. hal-00569832

\section{HAL Id: hal-00569832 \\ https://hal.science/hal-00569832}

Submitted on 25 Feb 2011

HAL is a multi-disciplinary open access archive for the deposit and dissemination of scientific research documents, whether they are published or not. The documents may come from teaching and research institutions in France or abroad, or from public or private research centers.
L'archive ouverte pluridisciplinaire HAL, est destinée au dépôt et à la diffusion de documents scientifiques de niveau recherche, publiés ou non, émanant des établissements d'enseignement et de recherche français ou étrangers, des laboratoires publics ou privés. 


\title{
Unidirectional SPP excitation at asymmetrical two-layered metal gratings
}

\author{
A Roszkiewicz* and W Nasalski \\ Institute of Fundamental Technological Research, Polish Academy of Science \\ Adolfa Pawińskiego 5b, 02-106 Warsaw, Poland \\ *E-mail: arosz@ippt.gov.pl
}

\begin{abstract}
A new configuration of a reflective one-dimensional surface-relief metallicgrating structure for unidirectional excitation of surface plasmon polaritons (SPPs) is proposed and numerically explored for plane wave normal incidence. The structure is embedded between two different dielectric media and composed of two layers, each of them consisted of periodically placed rectangular metal stripes. It is shown that even a small horizontal shift between these two layers, or a change of dielectric contrast of the grating fillings, may redirect energy flow propagation in a vicinity of the grating structure. From these two cases of counter propagating energy flows only one shows the field behaviour typical for SPP excitation. This evident directivity phenomenon exists together with high concentration of the electromagnetic field at the periodic structure. The configurations analysed may be useful in designing optical devices like photodetectors or, in general, in any case where efficient control of energy propagation directivity is of primary importance.
\end{abstract}

PACS: 73.20.Mf, 42.79.Dj

\section{Introduction}

Plasmonics is one of the most rapidly developing branches in modern science, with tremendous progress in all its theoretical and experimental aspects [1]. A great effort has been recently made in developing efficient methods for controlling surface plasmon polaritons (SPPs) [2] and designing new plasmonic devices with growing number of potential applications in several aspects of science and technology [3]. Those include, among others, spectroscopic chemical and biological sensors used in industrial process control, monitoring environmental pollution and planet surfaces, remote sensing with optical fibre sensors, as well as production of efficient solar cells and light-emitting diodes with high efficiency. Due to the strong wavelength discrimination effect, SPPs allow also producing wavelength-tunable filters. The ability of SPP to overcome the Rayleigh criterion together with great enhancement of electromagnetic field in near vicinity of a metal surface allows visualization of nanoobjects of dimensions much lower than an incident wavelength. In optical communication and data storage SPPs are considered as a new type of optical information carrier.

For further research and development of plasmonics, devising photonic elements similar to those used in conventional optics, but working with the help of SPPs, are necessary. For this goal, special photonic elements have been developed, like, for example, bent waveguides with high transmission efficiency, microgratings working as Bragg waveguides with orthogonal bends, beam splitters, Bragg mirrors, interferometers and SPP semiconductor lasers. In this paper we present another photonic structure which strongly interrelates its geometrical parameters with directivity of the SPP propagation. 


\section{Grating geometry and numerical modelling}

Surface plasmon polaritons, being electromagnetic waves coupled to density waves of free electrons in metals, are surface waves that propagate along the interface between two media with their dielectric constants of opposite signs [4]. They can be excited only with a TM polarized wave and, as having larger wave vector components in the horizontal direction than those of incident light, it happens only when this wave vector is increased in the direction of SPP propagation. Commonly used configurations for this purpose are glass prisms or diffraction gratings. In order to achieve SPP propagation in one desirable direction, an oblique incidence regime should be applied. However, in several configurations, due to complexity of the optical system, it is necessary to excite SPPs by normally incident beams. This also can be accomplished by using of diffraction gratings.

When light of frequency $\omega$ impinges on a one-dimensional (1D) diffraction grating at an angle $\theta_{\mathrm{i}}$, the horizontal (parallel to the grating vector) components of diffracted orders are: $\mathrm{k}_{\mathrm{x}}=\omega n \sin \left(\theta_{\mathrm{i}}\right) / \mathrm{c} \pm$ $\mathrm{mK}$, where $\mathrm{n}$ is the refractive index of the medium through which the grating is illuminated, $\mathrm{m}$ is an integer denoting the diffraction order and $c$ is the speed of light in vacuum, respectively. $K=2 \pi / \Lambda$ is reciprocal 1D lattice vector, where $\Lambda$ denotes the grating period. However, a significant waste of energy occurs when a symmetric grating is used due to equal coupling strengths of SPP propagating in the two opposite directions. This can be prevented by using a non-symmetric grating $[5,6,7]$. The authors of [5] analyzed two aluminium gratings. One was slanted rectangular grating and the second had smooth sinusoidal slanted shape. Although both gratings were shown to be able to efficiently lead SPPs in one direction, they are relatively difficult to manufacture, especially in the visible region, due to the requirement of maintaining high accuracy, e.g. in preserving slant angle.

Asymmetry of the SPP coupling is used also in designing directional beaming effects [8,9]. Offaxis directional beaming originates from different coupling efficiences of different gratings placed on both sides of a subwavelength slit in an optically thick metal film. By tuning gratings' parameters like a grating period in the case of a full-metallic grating or a refractive index in the case of a metallic grating covered by a dielectric layer, it is possible to adjust precisely an angle of the beam propagation direction. Similar breaking the configuration symmetry was utilized in designing slanted grating directional couplers [10]. Devices of such a type couple the light coming from a vertical fibre directly into a specially designed core and further, without any additional optics, into a horizontal waveguide.

We propose a different asymmetrical reflection configuration of the diffraction grating for unidirectional excitation of SPPs. By manipulating with asymmetry of the structure it is possible to change the direction of a local energy flow that is manifested by the sign change of the local Poynting vector near the surface. We will show that it is possible to achieve distinct and measurable effects resulted from the asymmetry of the periodic structure. The structure proposed in this paper could be manufactured with standard photolithography or focused ion beam milling processes. In this configuration the precise control of depth variation within the grating period is not so crucial as for the slanted gratings mentioned in [5]. In that case evaporation of subsequent layers must be repeated several times with different masks in order to approximate a desired smooth shape of the grating.

The problem under consideration is depicted in figure 1. Normally incident plane wave of wavelength $\lambda$ and TM polarization impinges on one-dimensional (1D) asymmetrical planar periodic structure. The structure is composed of two metal subgratings, one on the top of another and shifted in relation to each other. By $d_{x}$ we denote a shift between centres of metallic stripes in both gratings. A single grating layer is composed of periodic metal and dielectric areas. A complex valued refractive index of metal is described by Drude model:

$$
n_{2}(\omega)=\sqrt{1-\frac{\omega_{p}^{2}}{\omega^{2}+i \gamma \omega}}
$$

with parameters for gold: $\omega_{\mathrm{p}}=1.38 \cdot 10^{16} \mathrm{~s}^{-1}$ and $\gamma=1.07 \cdot 10^{14} \mathrm{~s}^{-1}$. Dielectrics placed between metal stripes in the upper and lower grating layers have the refractive indices $n_{4}$ and $n_{5}$, respectively. Dielectric substrate has the refractive index $\mathrm{n}_{3}$. The filling factors (areas occupied by metal in each layer) are denoted as $f_{1}$ and $f_{2}$, respectively and the period of the grating is $\Lambda$. The upper medium is air with $\mathrm{n}_{1}=1$, although may be replaced, in general, by any constant value $n_{1}$, when the ratio $n_{1} / n_{3}$ is preserved. Therefore, this configuration should be considered as the metal grating embedded between 
two dielectric media. The height of the whole grating structure is $\mathrm{h}$, the heights of individual grating layers are $h_{1}$ and $h_{2}$, respectively.

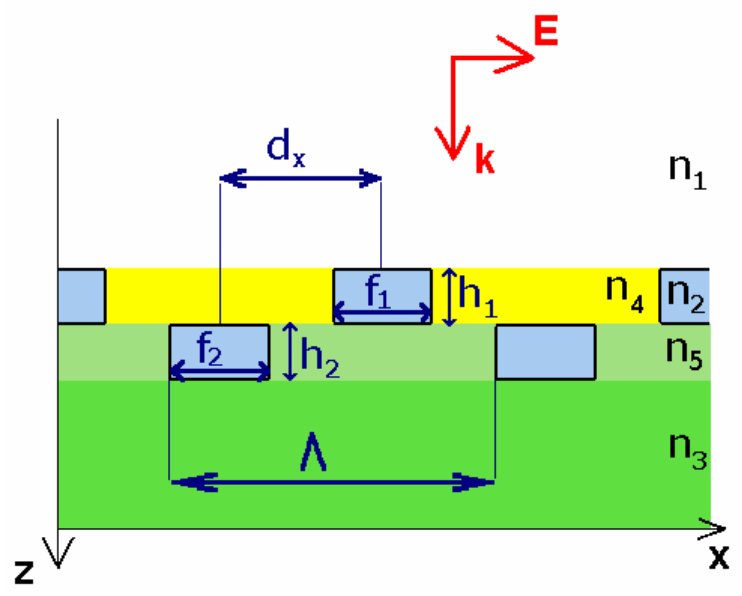

Figure 1 (colour online). Geometry of a two-layered grating diffraction problem. Two onedimensional surface-relief metallic diffraction gratings with different dielectric media in the gaps between the metal stripes are stacked and shifted in relation to each other.

In numerical calculations the Rigorous Coupled-Wave Analysis [11,12] (also called the Fourier Modal Method) with factorization rules described in [13] are applied. This frequency-domain method is widely used in grating diffraction analysis. It is based on the Fourier expansion of the relative permittivity function in the grating region. Maxwell equations in periodic region are solved for electric and magnetic fields decomposed into Floquet-Bloch modes. Eigenvalues and eigenmodes associated with grating modes are computed for each grating layer, invariant in the z-direction.

Those solutions are matched with each other and with solutions in the superstrate and the substrate by boundary conditions imposed on tangential field components. Eventually, the set of equations is solved for transmitted, reflected and grating mode amplitudes.

The energy flow (denoted by arrows on each fourth subplot of figures 2-5) is expressed by the time-averaged Poynting vector: $\langle\mathbf{S}\rangle=\operatorname{Re}\left(\mathbf{E} \times \mathbf{H}^{*}\right) / 2$, where $\mathbf{E}$ and $\mathbf{H}$ are electric and magnetic field vectors and $*$ denotes complex conjugation. The Poynting vector in each figure must be considered as a resultant energy flow of the incident wave and the reflected wave, where the reflected wave may acquire one from the two (positive or negative) propagation directions along the x-axis. The two waves propagating in the two opposite directions possess, in general, different complex amplitudes. A dispersive material (gold in our case) has the non-zero frequency-dependent absorption coefficient that increases significantly in abnormal dispersion regions. The relation between real and imaginary parts of susceptibility and the refractive index and the extinction coefficient are given by:

$$
n-i \frac{\gamma}{2 k_{0}}=\sqrt{1+\chi_{1}+i \chi_{2}}
$$

where $\mathrm{n}$ and $\gamma$ are the refractive index and the extinction coefficient, respectively, and $\mathrm{k}_{0}$ is a freespace wave-number. Kramers-Krönig relations relate $n$ and $\gamma$ for real $\left(\chi_{1}\right)$ and imaginary $\left(\chi_{2}\right)$ parts of susceptibility for a sufficiently wide range of frequencies.

In numerical simulations we assume $\lambda=632.8 \mathrm{~nm}, \mathrm{n}_{1}=1, \mathrm{n}_{3}=1.5, \Lambda=600 \mathrm{~nm}, \mathrm{~h}_{1}=\mathrm{h}_{2}=60 \mathrm{~nm}, \mathrm{f}_{1}$ $=\mathrm{f}_{2}$. The height of the grating should be precisely tailored in order to obtain the directionality effect, as will be described below. Values of refractive index of dielectric media are not considered as absolute values but rather as contrast values with respect to the upper dielectric medium. For simplicity we assume that $\mathrm{n}_{1}=1$ in the incident medium. Different configurations of the two-layer structure, with different directions of the field energy flow, will be presented in the next section. 


\section{Asymmetry of the grating configuration and field distribution}

The grating period is chosen smaller than the wavelength $\lambda$ in order to make all diffraction orders, except the zero-order one, evanescent with almost completely suppressed energy losses. When the reflectivity and transmittivity reach their minimum possible value, the electromagnetic field obtains its maximum value near the surface. In order to achieve the propagation of SPP in only one direction, amplitudes of specularly reflected waves have to be significantly decreased and the energy flow has to be transferred into the first diffraction order, due to the coupling of the one from many evanescent waves to SPP. Such a wave field should match the following conditions: the equality of frequency of the incident wave and SPP, as well as the equality of the incident wave vector horizontal component and the wave number of the SPP. We preliminary optimized the filling factors $f_{1}, f_{2}$ of both layers following the procedure similar to that described in [5]. The resultant filling factors are $f_{1}=f_{2}=0.4$. The fourth subplots of figures 2-5 shown below present the main result of numerical simulations, that is the magnitude and direction of the Poynting vector. The three remaining subplots displaying the electromagnetic field components $\mathrm{E}_{\mathrm{x}}, \mathrm{H}_{\mathrm{y}}$ and $\mathrm{E}_{\mathrm{z}}$, indicate the asymmetry of the field distributions.

\subsection{Influence of horizontal shifts in the grating geometry}

We first consider two structures characterized by the refractive indices $n_{4}=1$ and $n_{5}=1.5$, with the horizontal shift change between the upper and lower layers within the range between 0 and $0.5 \Lambda$. We plotted two configurations with different shifts: $d_{x}=0.2 \Lambda$ (figure 2 ) and $d_{x}=0.375 \Lambda$ (figure 3 ). In both figures the plane boundary between two dielectric fillings is placed in between two grating sublayers.

In both cases the asymmetrical distribution of the Poynting vector magnitude in the vicinity of the structure is clearly seen. There is a small leakage of radiation into the substrate and a distinct directionality of energy flow near the surface. The shift difference between both configurations is relatively small but, nonetheless, it changes completely the direction of energy propagation. Such a small shift difference causes a measurable change in the propagation conditions and therefore in the direction of energy propagation in the vicinity of the surface. It can be easily seen that the component of electromagnetic field that changes its sign at a certain value of $d_{x}$, reversing therefore the sign of the Poynting vector, is $\mathrm{H}_{\mathrm{y}}$. Resonance conditions at the upper and lower boundary are not equal due to the presence of media with different refractive indices and, therefore, the SPPs are excited only at one boundary.

Grating period has to be properly chosen with respect to the wavelength. Although it is not shown for brevity, we verified that too short or too large grating period with respect to the wavelength does not allow guiding the electromagnetic field along the structure in the form of SPP, since the coupling conditions between incident evanescent order and the SPP are not fulfilled. This means that for a given structure, even asymmetric, the choice of wavelength range is limited by the structure ability to change the direction of SPP propagation. Additionally, if grating is too thin or too thick, the directivity effect is also suppressed. This is the reason for choosing the height of the whole grating equal to $120 \mathrm{~nm}$. In particular, the values of half or twice the chosen height $(60 \mathrm{~nm}$ or $240 \mathrm{~nm})$ are too small or too large to enable relevant change in direction of energy propagation in the described configuration. Only the narrow range of grating heights allows achieving the opposite energy flow directions for the shifts between $0 \leq \mathrm{d}_{\mathrm{x}} \leq 0.5 \Lambda$.

There are also some general restrictions regarding the filling factor. It cannot be too small in order to prevent direct transmission of light between the metal stripes into the substrate. On the other side, we have also numerically verified that too wide metal stripes cause creation of additional curls above the grating, due to reflection of significant amount of electromagnetic field from metal areas. The reflected field interferes with the incident light and form an interference pattern. This phenomenon smears out the directionality and smoothness of the energy flow. 

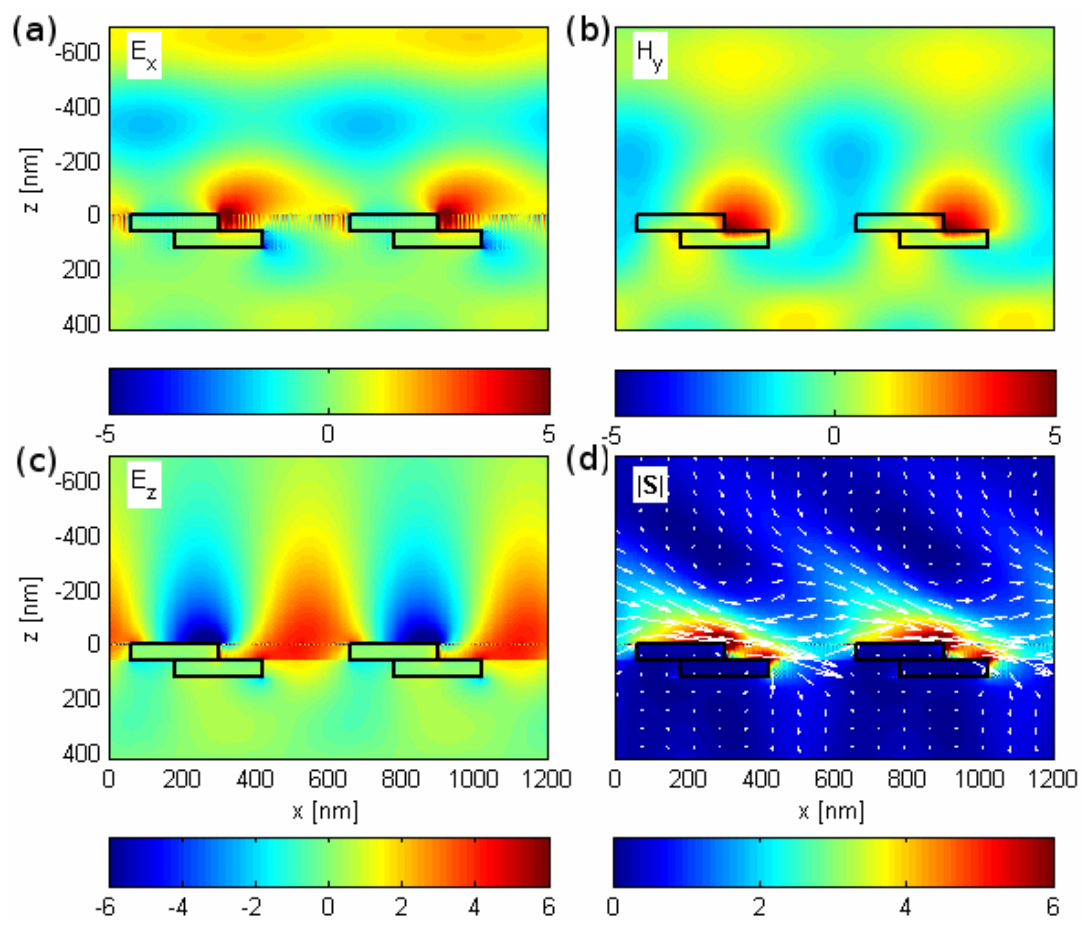

Figure 2 (colour online). Grating configuration with $\mathrm{n}_{4}=1, \mathrm{n}_{5}=1.5$ and $\mathrm{d}_{\mathrm{x}}=0.2 \Lambda$ and all nonzero components of electromagnetic field, normalized to the incident wave, in the vicinity of diffraction grating: (a) $\mathrm{E}_{\mathrm{x}}$, (b) $\mathrm{H}_{\mathrm{y}}$ and (c) $\mathrm{E}_{\mathrm{z}}$ and (d) the Poynting vector magnitude $|\mathbf{S}|$ with the direction of the energy flow denoted by arrows.
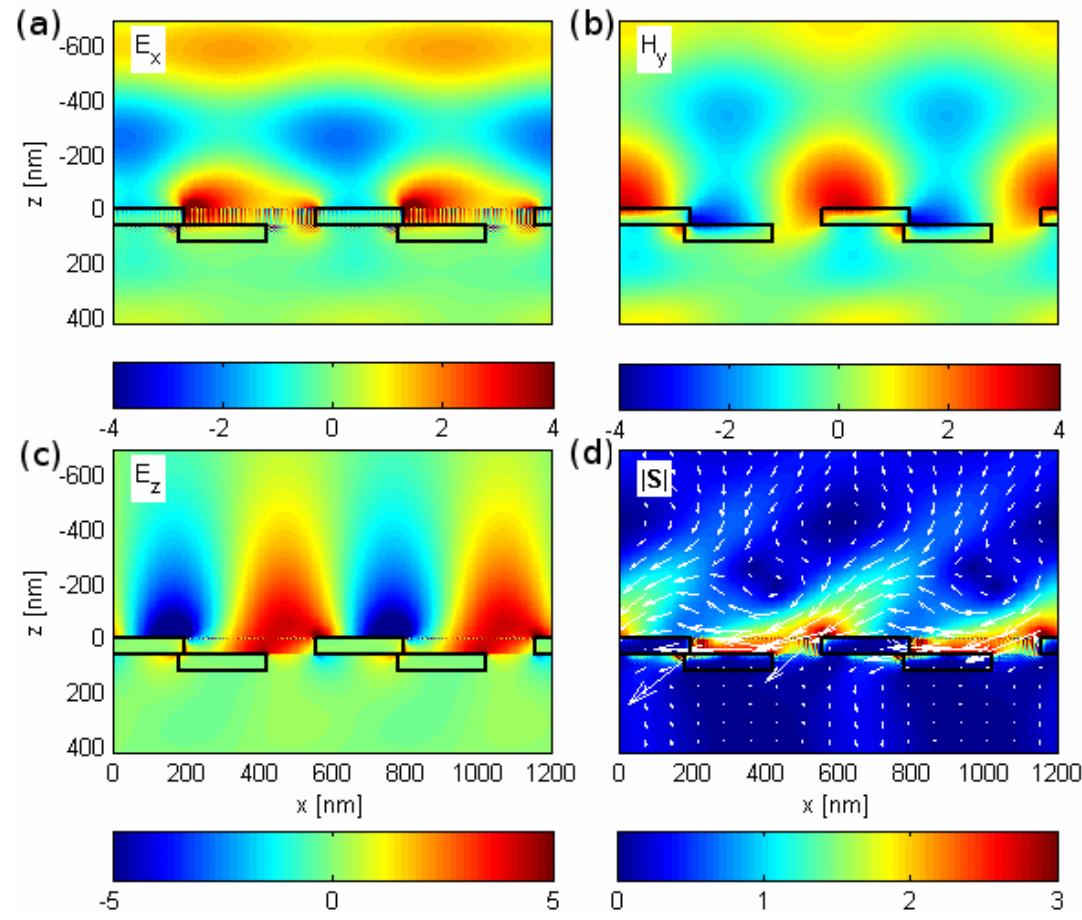

Figure 3 (colour online). Grating configuration with $n_{4}=1, n_{5}=1.5$ and $d_{x}=0.375 \Lambda$ and all nonzero components of electromagnetic field, normalized to the incident wave, in the vicinity of diffraction grating: (a) $\mathrm{E}_{\mathrm{x}}$, (b) $\mathrm{H}_{\mathrm{y}}$ and (c) $\mathrm{E}_{\mathrm{z}}$ and (d) the Poynting vector magnitude $|\mathbf{S}|$ with the direction of the energy flow denoted by arrows. 


\subsection{Influence of the dielectric medium inside the grating regions}

Subsequently, we analyze the field behaviour above the structure specified by a vertical shift of the dielectric boundary inside the grating region. Two cases are presented. In the first case, the grating filled with dielectric of the refractive index higher than incident medium $n_{4}=n_{5}>n_{1}$ is considered (figure 4). In the second case, the grating with dielectric medium of the refractive index equal to the index of incident medium $n_{4}=n_{5}=n_{1}$ is presented (figure 5). In both cases it is possible to find a particular value of horizontal shift $\left(\mathrm{d}_{\mathrm{x}}\right)$ at which the strong directional effect is obtained and significant asymmetry in the field distributions occurs. Most of the field energy density, flows along the surface. Disturbances above the structure are small and the flow over the grating is not very much disturbed. In this case the change of field energy directivity is imposed by the vertical shift of dielectric media, not by the horizontal mutual shift $\left(\mathrm{d}_{\mathrm{x}}\right)$ of metallic stripes, as it was shown in the previous subsection.

An interesting comparison can be made when one structure is filled with different dielectric media in both periodic layers and the second structure of the same fillings in both periodic layers. The presence of the dielectric of the higher refractive index changes radically the direction of the energy flow in comparison with the structure filled with completely the same dielectric medium, certainly in spite of the substrate. In the metallic structure exposed to dielectric with $\mathrm{n}_{4}=\mathrm{n}_{5}=\mathrm{n}_{1}=1$ the energy propagation direction is the same as in case of slanted metallic grating placed on a metal substrate [5]. However, by adding a higher refractive index material between the metal stripes the sign of the Poynting vector changes and the energy flow becomes oppositely directed. The difference in those cases is, as in the previous cases, connected with change of sign of the $\mathrm{H}_{\mathrm{y}}$ component.
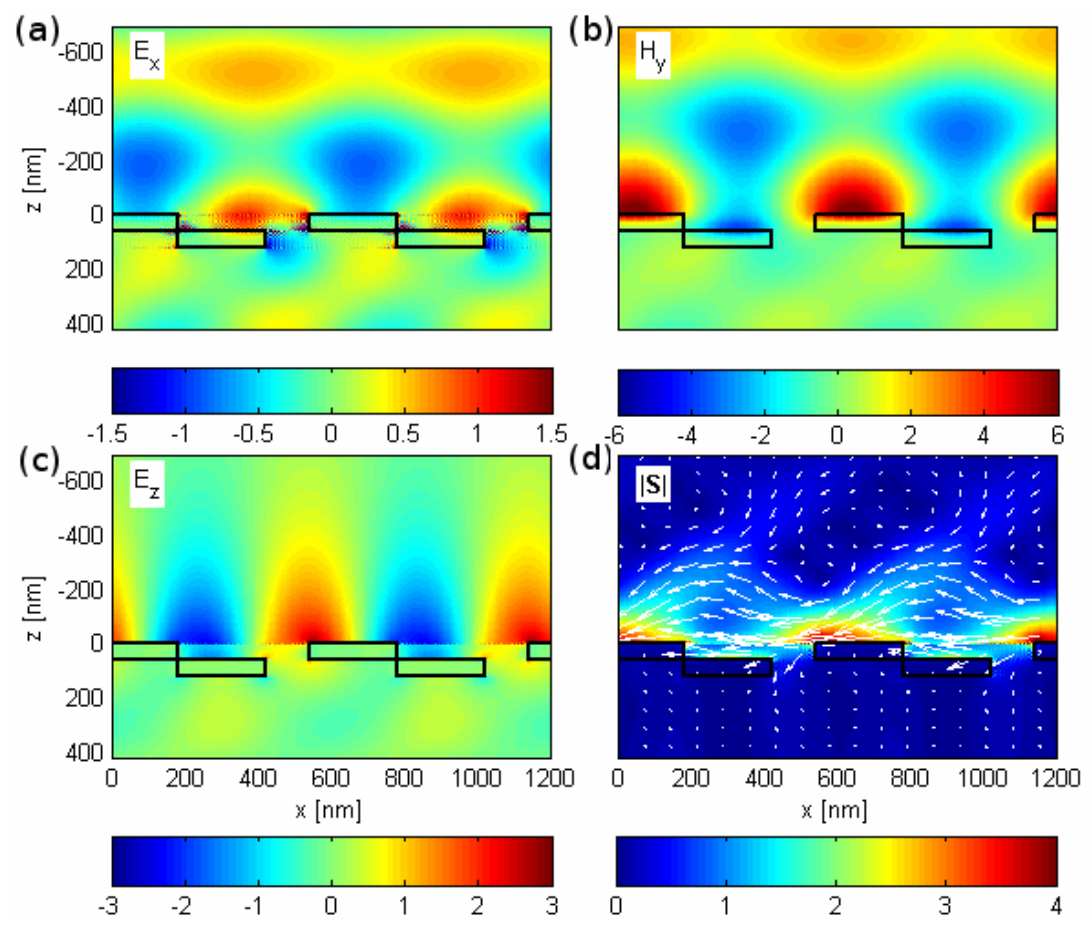

Figure 4 (colour online). Grating configuration with $\mathrm{n}_{4}=1.5, \mathrm{n}_{5}=1.5$ and $\mathrm{d}_{\mathrm{x}}=0.4 \Lambda$ and all nonzero components of electromagnetic field, normalized to the incident wave, in the vicinity of diffraction grating: (a) $\mathrm{E}_{\mathrm{x}}$, (b) $\mathrm{H}_{\mathrm{y}}$ and (c) $\mathrm{E}_{\mathrm{z}}$ and (d) the Poynting vector magnitude $|\mathbf{S}|$ with the direction of the energy flow denoted by arrows. 

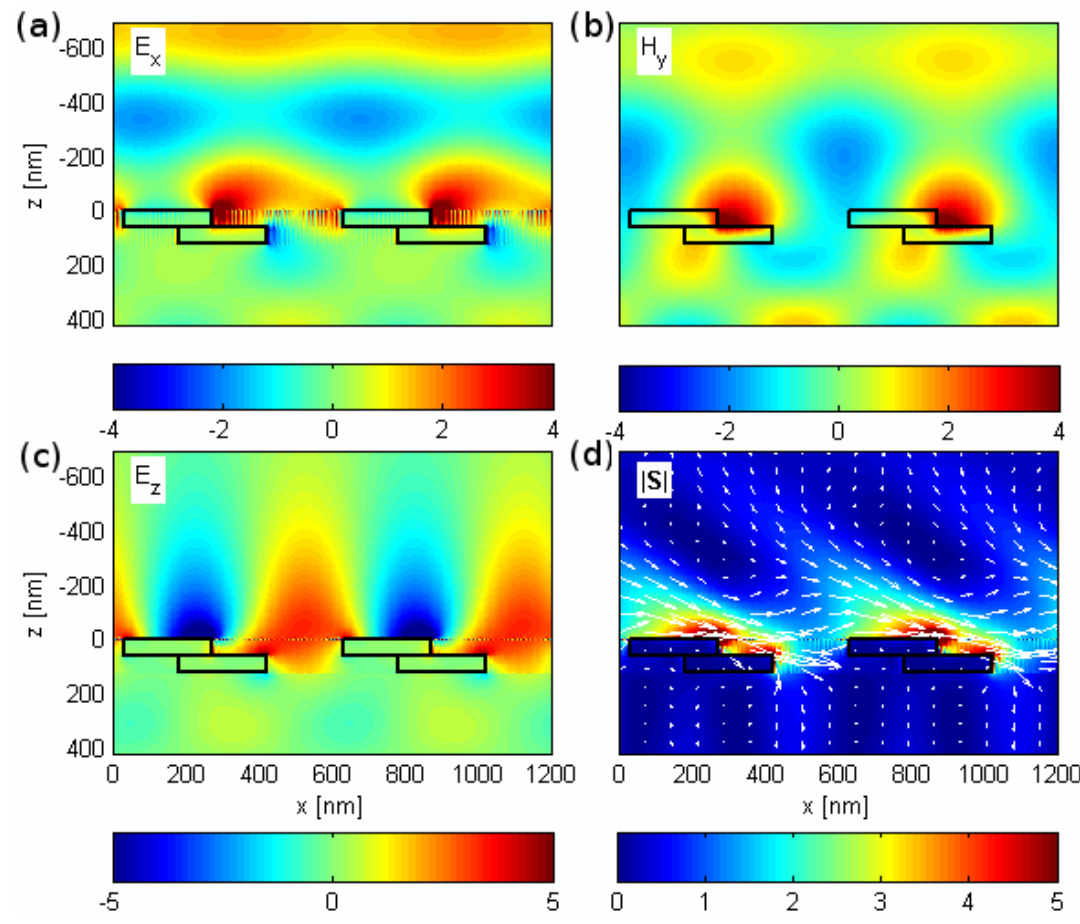

Figure 5 (colour online). Grating configuration with $\mathrm{n}_{4}=1, \mathrm{n}_{5}=1$ and $\mathrm{d}_{\mathrm{x}}=0.25 \Lambda$ and all nonzero components of electromagnetic field, normalized to the incident wave, in the vicinity of diffraction grating: (a) $\mathrm{E}_{\mathrm{x}}$, (b) $\mathrm{H}_{\mathrm{y}}$ and (c) $\mathrm{E}_{\mathrm{z}}$ and (d) the Poynting vector magnitude $|\mathbf{S}|$ with the direction of the energy flow denoted by arrows.

It seems that the difference between the refractive indices in the grating layers $n_{4}$ and $n_{5}$ is crucial for obtaining the one from the two opposite directions of the energy flow in the two, nearly the same, configurations. They differ only slightly in $d_{x}$. The analysis of the $E_{x}$ and $H_{y}$ components shows that the field is tightly confined in the vicinity of the metal stripes, and therefore is stronger in cases when the stripes are embedded in dielectrics with $n_{4}, n_{5}>1$, in opposition to the case of $n_{4}=n_{5}=n_{1}=1$. Propagation constants of the SPPs moving in the opposite directions (+/- first diffraction orders) are equal in the case of normal incidence, despite of the asymmetry of the grating. It appears that decisive in the unidirectional propagation of the SPPs are the different coupling strengths of both SPPs and, in consequence, different amplitudes of the both propagating modes [5].

\subsection{Action of the structures with horizontal symmetry}

Finally, we present two grating configurations that are symmetrical with respect to the $y-z$ plane (figure 6), that is the structures specified by $d_{x}=0.5 \Lambda$. The refractive indices in the first configuration are: $\mathrm{n}_{4}=1, \mathrm{n}_{5}=1.5$, in opposition to the second one: $\mathrm{n}_{4}=\mathrm{n}_{5}=1.5$. In both configurations the curls, in centres of which the energy flow is zero, emerge in the distribution of the Poynting vector. Those curls are originating from the interference of incoming, reflected and diffracted fields, together with the propagating SPPs. This interference is destructive and decreases the magnitude of the Poynting vector. 

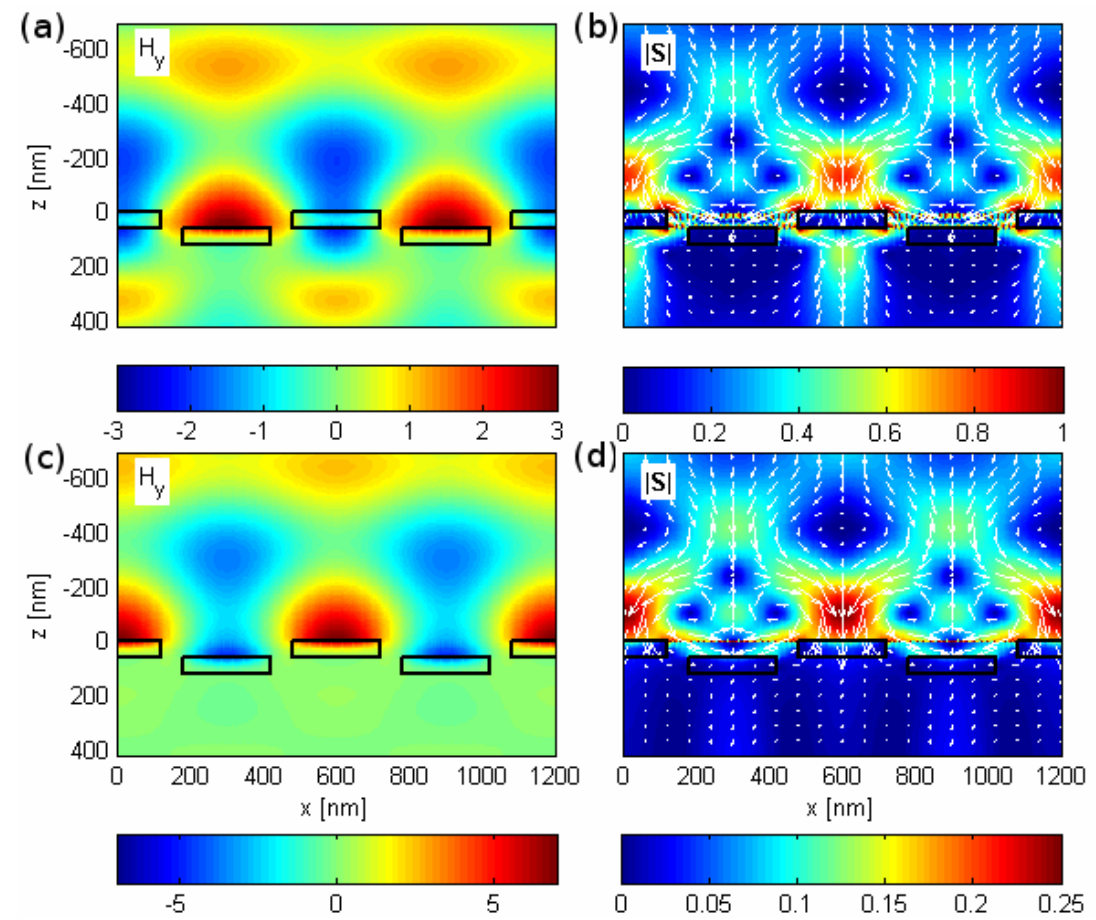

Figure 6 (colour online). Two symmetrical grating configurations $\left(d_{x}=0.5 \Lambda\right)$ with $n_{4}=1, n_{5}=1.5$ (upper subplots (a) and (b)) and $\mathrm{n}_{4}=\mathrm{n}_{5}=1.5$ (lower subplots (c) and (d)) and the component of the magnetic field $\mathrm{H}_{\mathrm{y}}$ (left column (a) and (c)) and the Poynting vector magnitude $|\mathbf{S}|$ with the direction of the energy flow denoted by arrows (right column (b) and (d)).

As the structure is symmetrical with respect to the $y-z$ plane, no direction along $x$-axis is preferred and no horizontal energy flow near the grating structure is observed. Moreover, the replacement of $n_{4}$ in the first configuration by $n_{1}$ in the second one, changes the sign of the magnetic field component $\mathrm{H}_{y}$, but still maintains the shape of the interference pattern over the whole structure.

\subsection{Additional remarks}

The results presented above have been discussed in the context of the energy flow direction of the total field. However, apparently similar or almost symmetric results shown for the Poynting vector evaluated over the really asymmetric grating structure may suggest different physical phenomena, which are the origins of the field propagation in the two opposite directions. In order to investigate physical reasons of such behaviour of asymmetric two-layered structure, we plot in addition the local reflection coefficient for one of the mentioned above geometries of the structure. Similar charts could be plotted for other configurations, although, for clarity, we present here only one set of the plots for one configuration.

The plots in figure 7 are calculated for the case of the material and geometrical parameters related to these of figures 2 and 3 (i.e. $\mathrm{n}_{4}=1, \mathrm{n}_{5}=1.5$ ). They present the local reflection coefficients of the +1 diffraction order, the -1 diffraction order and all diffraction orders together for $\mathrm{E}_{\mathrm{x}}$ and $\mathrm{H}_{\mathrm{y}}$ components of electromagnetic field. For this purpose the local reflection coefficient is defined at some point above the structure as the total field reduced by the incident field and normalized to the incident field. We plotted the local reflection coefficients just above the structure, at the points $2 \mathrm{~nm}$ over the middle of upper metal stripes, where the energy flow has the smoothest profile. These plots refer to numerical simulations illustrating the change of the Poynting vector sign in the presence of small structure modifications.

Figure 7 indicates that the geometry of the structure really favours the SPP excitation only in one direction. However, this probably does not exclude the change of Poynting vector sign and therefore 
energy propagation in the opposite direction without the excitation of SPP. Our results indicate the presence of two different kinds of phenomena which give analogous results in the context of the directivity of the energy flow. Strong discontinuities of the local reflection coefficient in the case of horizontal shift slightly below $d_{x}=0.4 \Lambda$ relate the resonance conditions for unidirectional SPP excitation. The change of directivity of energy flow near the value of $d_{x}=0.2 \Lambda$ is connected with other physical phenomena, probably the diffraction effects farther from the SPP resonance. This assumption should be verified in further research.
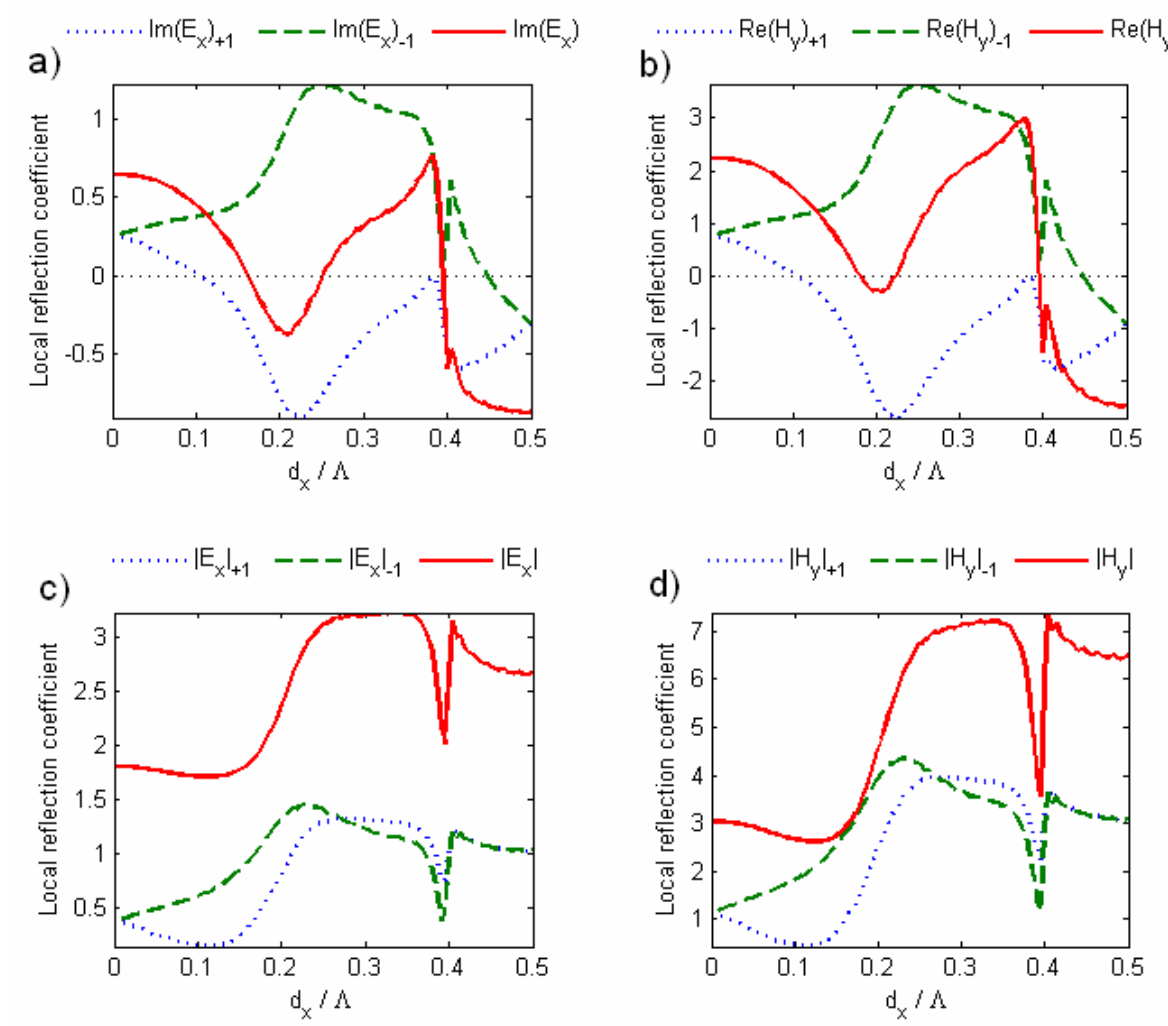

Figure 7 (colour online). Local reflection coefficient plotted versus the horizontal shift $d_{x}$ for the grating configuration as in figures 2 and $3\left(\mathrm{n}_{4}=1, \mathrm{n}_{5}=1.5\right)$; an imaginary part (a) and a magnitude (c) of $E_{x}$, together with a real part (b) and a magnitude (d) of $\mathrm{H}_{\mathrm{y}}$, of the local reflection coefficient of +1 diffraction order (blue dotted line), -1 diffraction order (green dashed line) and of all diffraction orders (red solid line).

It appears that in cases of too thin or too thick metal stripes the energy flow redirection cannot be achieved. Suppression of the directivity effect in the case of too thin gratings may be explained by the weak influence of thin metal stripes on the incident wave and the quite high transmittivity of the structure. In this case the metal stripes are not sufficiently optically thick and opaque. On the other hand, in the case of too thick gratings, the influence of the lower subgrating on the incident field may be too weak to enable the efficient redirection of the energy flow.

The explanation of the numerical results is given here on the grounds of the examples of the plane wave incidence upon the infinite reflecting structures. Even this idealized geometry allows us to achieve a few interesting observations. They indicate that the change of the energy flow direction is possible by introduction of small differences either in the structure geometry or in values of the refractive index of the dielectric filling. The excitation efficiency of this energy flow redirection can be estimated in this idealized view of this phenomenon as well, as it is shown in figure 8. In this figure the normalized (to the incident field amplitude) amplitudes of the reflected from and transmitted through the structure optical field are drawn. The differences between the left and the right field peaks 
are consistent with the direction of the energy flow preferred in the two cases described in figures 2 and 3 and subsequently in figure 7 .
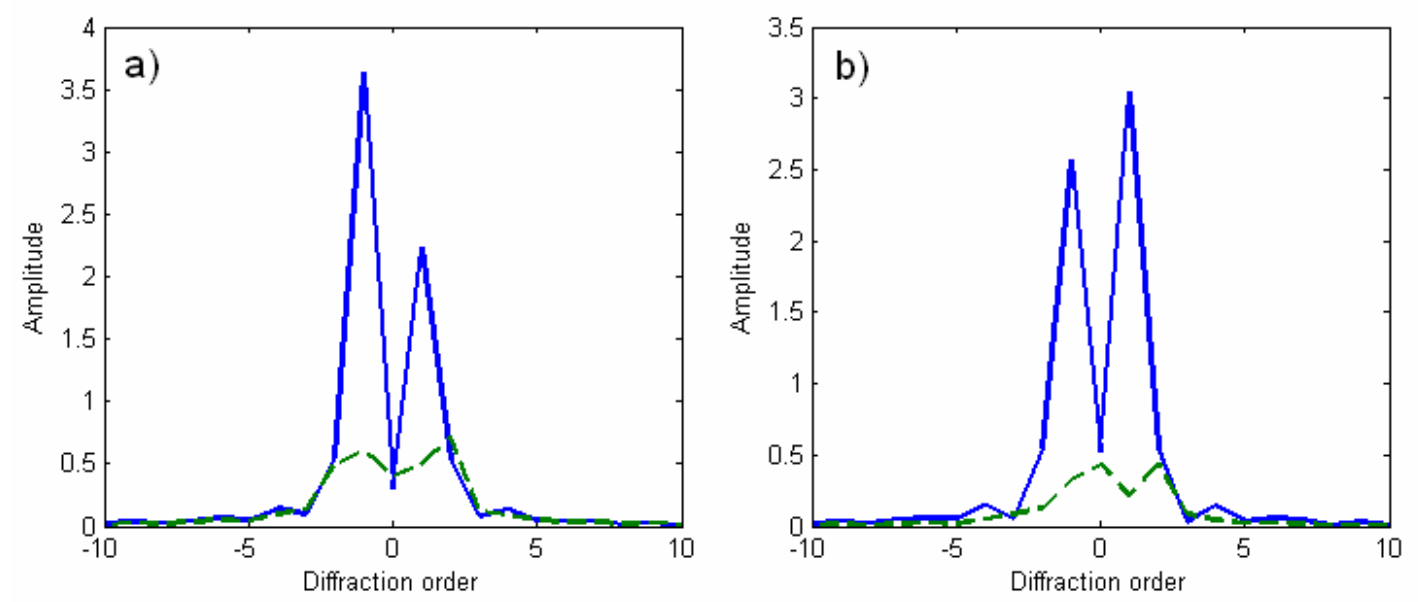

Figure 8 (colour online). Normalized amplitudes of the reflected (blue solid line) and transmitted (green dashed line) optical field measured above and below the two-layered structure for the shift $\mathrm{d}_{\mathrm{x}}=0.2 \Lambda$ and the energy flow in the $+\mathrm{x}$ direction (a) and for $\mathrm{d}_{\mathrm{x}}=0.375 \Lambda$ and the energy flow in the $-\mathrm{x}$ direction (b).

Further analysis and more complete interpretation of these phenomena will be possible by taking into account a more realistic case of finite geometry of the problem. This means the cases of beams with finite cross-sections incident on spatially finite periodic SPPs guiding structures. In such cases we will be able to take into account the finite excitation area of SPPs, precisely calculate their excitation efficiency, propagation length and other quantitative parameters specifying more realistically the problem under consideration.

\section{Conclusion}

We have demonstrated that a simple two-layered structure, composed of two gratings with rectangular metal stripes embedded in dielectric media, can be used to control electromagnetic field behaviour in the vicinity of this structure. By a specific choice of the geometric configuration of the structure one may determine a desired direction of the energy flow. It appears that, by tailoring properly the horizontal asymmetry of the structure by shifting the gratings with respect to each other, it is possible to redirect the energy flow into one of the two opposite directions. Moreover, the grating shifts applied for this purpose may be small. The directivity change of the energy flow direction is accompanied by a sign change of the magnetic field component $\mathrm{H}_{\mathrm{y}}$ at a certain value of the shift $\mathrm{d}_{\mathrm{x}}$. The same directivity change can be also obtained at a specified vertical position of a contrast boundary between two dielectric filling media. The advantage of this configuration is that it is easy to produce with the photolithography or focused ion beam techniques, which are not time or cost consuming. Moreover, in the case of metal stripes embedded in the two different dielectrics, the field components are tightly confined to the metal structure.

The geometry analyzed may find several applications in photonics. It can serve as an optical device for controlling direction of electromagnetic energy flow. Maintaining the advantages of normal incidence, it opens, at the same time, the possibility of manipulation with the direction of energy propagation. Similar simple asymmetric grating configurations with further optimization can serve as optical concentrators. By breaking the symmetry of the grating and thus forming a subwavelength slit between two grating systems with the reversed shift, and properly adjusting the phase differences of SPPs incoming from both sides, a strong concentration of light in the middle of the structure can be obtained. Such configurations can serve as elements of photodetectors, where the radiation has to be concentrated within a small active area below the metallization region. 


\section{References}

[1] Girard C 2005 Near fields in nanostructures Rep. Prog. Phys. 68 1883-1933

[2] Lee B, Kim S, Kim H and Lim Y 2010 The use of plasmonics in light beaming and focusing Progress in Quantum Electronics 34 47-87

[3] Roszkiewicz A, Szabelak W and Nasalski W 2009 Surface plasmon polariton applications in selected branches of modern science and technology J.Tech. Phys. 50 1, 3-16

[4] Raether R 1988 Surface Plasmons on Smooth and Rough Surfaces and on Gratings (Berlin, SpringerVerlag)

[5] Bonod N, Popov E, Li L and Chernov B 2007 Unidirectional excitation of surface plasmons by slanted gratings Opt. Ex. 15 11427-32

[6] Kleemann B H, Ruoff J and Arnold R 2005 Area-coded effective medium structures, a new type of grating design Opt. Lett. 30, 1617-9

[7] Bai B, Meng X, Laukkanen J, Sfez T, Yu L, Nakagawa W, Herzig H P, Li L and Turunen J 2009 Asymmetrical excitation of surface plasmon polaritons on blazed gratings at normal incidence Phys. Rev. B 80035407

[8] Kim S, Kim H, Lim Y and Lee B 2007 Off-axis directional beaming of optical field diffracted by a single subwavelength metal slit with asymmetric dielectric surface gratings Appl. Phys. Lett. 90, 051113

[9] Kim H, Park J and Lee B 2009 Tunable directional beaming from subwavelength metal slits with metal-dielectric composite surface gratings Opt. Lett. 34, 2569-71

[10] Wang B, Jiang J and Nordin G P 2004 Compact slanted grating couplers Opt. Express 12, 3313-26

[11] Moharam M G, Grann E B, Pommet D A and Gaylord T K 1995 Formulation for stable and efficient implementation of the rigorous coupled-wave analysis of binary gratings J. Opt. Soc. Am. A 12 1068-76

[12] Moharam M G, Pommet D A., Grann E B and Gaylord T K 1995 Stable implementation of the rigorous coupled-wave analysis for surface-relief gratings: enhanced transmittance matrix approach Opt. Soc. Am. A 12 1077-86

[13] Li L 1996 Use of Fourier series in the analysis of discontinuous periodic structures J. Opt. Soc. Am. A 13 1870-6 